ITEP-TH-41/99

\title{
Short strings and gluon propagator in the infrared region.
}

\author{
M.N. Chernodub ${ }^{\mathrm{a}}$, F.V. Gubarev ${ }^{\mathrm{a}}$, M.I. Polikarpov ${ }^{\mathrm{a}}$, V.I. Zakharov ${ }^{\mathrm{b} *}$ \\ anstitute of Theoretical and Experimental Physics, \\ B.Cheremushkinskaya 25, Moscow, 117259, Russia \\ ${ }^{b}$ Max-Planck Institut für Physik, \\ Föhringer Ring 6, 80805 München, Germany
}

We discuss how infrared region influence on short distance physics via new object, called "short string". This object exists in confining theories and violates the operator product expansion. Most analytical results are obtained for the dual Abelian Higgs theory, while phenomenological arguments are given for QCD.

\section{Introduction}

Below we describe effects produced by the recently introduced object, short string. Short strings [1] are responsible for non-standard power corrections in QCD, which are important in the ultraviolet region. On the other hand short strings exist due to confinement and in this sense they are induced by large distance physics. Short strings are not point-like objects and violate such fundamental principle as operator product expansion (OPE).

It is interesting to find the short string effects in QCD, but confinement in QCD can not be described from first principles and at the beginning (in Sects. 2-4) we consider the dual Abelian Higgs model (AHM). In this model electric charges are confined at the classical level, and it is simple enough to study both large and small distances. Moreover, due to the Abelian dominance [2], the dual AHM can be considered as the effective infrared theory for QCD. At small distances dual AHM is not related to QCD, and we consider it to study general properties of short strings. We show that short strings give rise to special singularities in the photon propagator and produce the linear correction, $\sigma_{0} r$, to the Coulomb potential, $\alpha / r$, at small distances.

In Sect. 5 we discuss numerical (lattice calculations) and phenomenological facts supporting the

\footnotetext{
*Material based on talks by F.V. Gubarev and M.I. Polikarpov at Lattice '99, Pisa, Italy.
}

existence of short strings in QCD.

\section{Short strings in classical equations of motion for AHM}

Consider the following problem: what are corrections to Coulomb potential at small distances in QCD? If we neglect the trivial perturbative dependence of the bare coupling on $r$, we have in the standard approach (perturbation theory $+\mathrm{OPE}$ ): $V(r)=-\alpha / r+c \cdot r^{\gamma}$, and $\gamma=2$ or 3 depending on the parameters of the system (see [3, 4 i).

Consider now the dual AHM with the action:

$$
\begin{aligned}
S=\int d^{4} x\left[\frac{1}{4 g^{2}} F_{\mu \nu}^{2}\right. & +\frac{1}{2}|(\partial-i B) \Phi|^{2}+ \\
& \left.+\frac{1}{4} \lambda\left(|\Phi|^{2}-\eta^{2}\right)^{2}\right],
\end{aligned}
$$

where $B$ is the dual gauge field, $\Phi$ is the Higgs field carrying the magnetic charge $g$. The Higgs field and the gauge field are massive: $m_{H}^{2}=$ $2 \lambda \eta^{2}, m_{V}^{2}=g^{2} \eta^{2}$. The classical potential between $+e$ and $-e$ charges (analogue of the quark - antiquark pair) at large distances is:

$V(r)=\sigma_{\infty} \cdot r \quad(r \rightarrow \infty)$

The confining behavior is due to the dual Abrikosov - Nielsen -Olesen (ANO) string which is formed between $+e$ and $-e$ charges. At small distances the potential is Coulomb like: $V(r)=$ $-\alpha / r, r \rightarrow 0$. We are interested in the classical corrections to the Coulomb potential at $r \ll$ 
$m_{H}^{-1}, m_{V}^{-1}$ [1]. One has to impose the boundary condition, that is the vanishing of the scalar field along a mathematically thin line connecting the magnetic charges. This topological condition was formulated in Ref. [5] and implied in numerical studies of the problem (see, e.g., [6]).

This infinitely thin line is nothing else but the Dirac string connecting the external charges. By definition the energy of the Dirac string is zero. It is easy to realize that the Dirac string cannot coexist with $\Phi \neq 0$. Indeed, if the Dirac string would be embedded into a vacuum with $\langle\Phi\rangle \neq 0$ then its energy would again jump to infinity since there is the term $1 / 2|\Phi|^{2} A_{\mu}^{2}$ in the action and $A_{\mu}^{2} \rightarrow \infty$ for a Dirac string. Therefore, even in the limit $r \rightarrow 0$ there is a deep well in the profile of the Higgs field $\Phi$. This might cost energy which is linear with $r$ even at small $r$.

Now we are coming to the next question, whether this mathematically thin line realizes as a short physical string. By the physical string we understand a stringy piece in the potential, $\sigma_{0} \cdot r$ at small $r$. In other words, we are going to see whether the stringy boundary condition implies a stringy potential. To get the answer one solves the classical equations of motion, the result is [1]:

$\lim _{r \rightarrow 0} V(r)=-\frac{\alpha_{M}}{r}+\sigma_{0} \cdot r+\ldots$

with a non-vanishing slope $\sigma_{0}$.

The slope $\sigma_{0}$ depends smoothly on the value of $m_{V} / m_{H}$. For example for $m_{V}=m_{H}$ the slope of the potential at $r \rightarrow 0$ is the same as at $r \rightarrow \infty$. That is, within error bars:

$\sigma_{0} \approx \sigma_{\infty}$

where $\sigma_{\infty}$ determines the value of the potential at large $r$, (2).

\section{Gauge boson propagator in the confin- ing theory}

The simplest "phenomenological" theory of the quark confinement is formulated in terms of the gluon propagator. Namely, one argues [7, [] that if the gluon propagator has a double pole in the infrared region then the quark-antiquark potential gets a linear attractive contribution at large distances and this implies the quark confinement. On the other hand, Gribov and Zwanziger [9] argued that elimination of the gauge copies leads to a counter-intuitive vanishing of the gluon propagator in the infrared region. Some analytical studies 10 confirm this behavior, while the others 11] predict a singular gluon propagator in the infrared limit. Recent numerical simulations in lattice gluodynamics [12 show that the gluon propagator seems to be finite at small momenta.

Below we consider the gauge boson propagator in dual AHM, that is the abelian analogue of the gluon propagator. To simplify calculations we consider the London limit, that is $\lambda \rightarrow \infty$ in (11), and $m_{H} \rightarrow \infty$. The final result remains unchanged if we relax the condition on the coupling $\lambda$. To evaluate the propagator we utilize the Zwanziger local field theory of electrically and magnetically charged particles 13]. The theory contains two vectors potentials, namely the gauge field $A_{\mu}(x)$ and the dual gauge field $B_{\mu}(x)$, which interact covariantly with electric and magnetic currents, respectively. The corresponding Lagrangian is:

$$
\begin{aligned}
\mathcal{L}=\mathcal{L}_{Z w}(A, B)+i e j_{\mu}^{e}(x) & A_{\mu}(x)+ \\
& +i g j_{\mu}^{g}(x) B_{\mu}(x),
\end{aligned}
$$

where $e(g)$ stands for the electric (magnetic) charge, and $j_{\mu}^{e}\left(j_{\mu}^{g}\right)$ is the electric (magnetic) external current. The Zwanziger Lagrangian $\mathcal{L}_{Z w}$ is 13]:

$$
\begin{aligned}
\mathcal{L}_{Z w}=\frac{1}{2}( & \cdot[\partial \wedge A])^{2}+\frac{1}{2}(n \cdot[\partial \wedge B])^{2}+ \\
+ & \frac{i}{2}(n \cdot[\partial \wedge A])\left(n \cdot[\partial \wedge B]^{d}\right)- \\
& -\frac{i}{2}(n \cdot[\partial \wedge B])\left(n \cdot[\partial \wedge A]^{d}\right),
\end{aligned}
$$

where $[A \wedge B]_{\mu \nu}=A_{\mu} B_{\nu}-A_{\nu} B_{\mu}, \quad(n \cdot[A \wedge$ $B])_{\mu}=n_{\nu}(A \wedge B)_{\nu \mu}, \quad(G)_{\mu \nu}^{d}=\frac{1}{2} \varepsilon_{\mu \nu \lambda \rho} G_{\lambda \rho}$. Inspite the Lagrangian (5) contains two gauge fields, $A_{\mu}$ and $B_{\mu}$, there is only one physical particle (a massless photon) in the spectrum 13, 14. The Lagrangian (5) depends on an arbitrary constant unit vector $n_{\mu}, n_{\mu}^{2}=1$, while physical observables are insensitive to the direction of $n$ provided the Dirac quantization condition, $e \cdot g=2 \pi m, m \in \mathbb{Z}$, is satisfied [13]. 
The theory of condensed monopoles interacting with an external electric source $j^{e}$ (quark current) is described by the following partition function [15]:

$$
\begin{gathered}
\mathcal{Z}\left[j^{e}\right]=\int \mathcal{D} A \mathcal{D} B \mathcal{D} \Phi \exp \left\{-\int \mathrm{d}^{4} x\left(\mathcal{L}_{Z w}(A, B)\right.\right. \\
\left.\left.+\frac{1}{2}|(\partial+i g B) \Phi|^{2}+\lambda\left(|\Phi|^{2}-\eta^{2}\right)^{2}-i e j^{e} A\right)\right\} .
\end{gathered}
$$

We work in the Euclidean space and consider the London limit, $\lambda \rightarrow \infty$. The gauge field propagator is defined as:

$$
D_{\mu \nu}(x, y)=-\left.\frac{1}{e^{2}} \frac{\delta^{2}}{\delta j_{\mu}^{e}(x) \delta j_{\nu}^{e}(y)} \frac{\mathcal{Z}\left[j^{e}\right]}{\mathcal{Z}[0]}\right|_{j^{e}=0}
$$

To specify the propagator completely we choose the axial gauge $n_{\mu} A_{\mu}=0$. The standard parameterization of the propagator in this gauge is 8 :

$$
\begin{gathered}
D_{\mu \nu}^{\text {axial }}=\left(\delta_{\mu \nu}+\frac{p_{\mu} n_{\nu}+p_{\nu} n_{\mu}}{(p \cdot n)}-\frac{p_{\mu} p_{\nu}}{(p \cdot n)^{2}}\right) F\left(p^{2}\right)- \\
-\frac{1}{(p \cdot n)^{2}} \cdot\left(\delta_{\mu \nu}-n_{\mu} n_{\nu}\right) G\left(p^{2}\right) .
\end{gathered}
$$

Using methods of Refs. [16] the functions $F\left(p^{2}\right)$ and $G\left(p^{2}\right)$ can be expressed through the ANO string-string correlator 17]:

$$
\begin{array}{r}
F\left(p^{2}\right)=\frac{1}{p^{2}+m_{V}^{2}}\left(1+\frac{m_{V}^{4} D^{\Sigma}\left(p^{2}\right)}{p^{2}+m_{V}^{2}}\right) \\
G\left(p^{2}\right)=-\frac{m_{V}^{2}}{p^{2}+m_{V}^{2}}\left(1-m_{V}^{2} \frac{p^{2} D^{\Sigma}\left(p^{2}\right)}{p^{2}+m_{V}^{2}}\right),
\end{array}
$$

where

$$
\begin{aligned}
& D_{\mu \nu}^{\Sigma}(p)=-\frac{\eta^{4} e^{2}}{\left(p^{2}+m_{V}^{2}\right)(p \cdot n)} . \\
& \quad \cdot \int \frac{\mathrm{d}^{4} k}{(2 \pi)^{2}} \frac{n_{\alpha} n_{\beta}<\Sigma_{\mu \alpha}(p) \Sigma_{\nu \beta}(-k)>_{\Sigma}}{\left(k^{2}+m_{V}^{2}\right)(k \cdot n)},
\end{aligned}
$$

and the correlation function of ANO strings $<\Sigma_{\mu \alpha}(p) \Sigma_{\nu \beta}(-k)>$ is formally defined in [16].

The propagator (8) has singularities not only in the $p^{2}$ plane but in the variable $(p \cdot n)$ as well. The singular in $(p \cdot n)$ terms appear not only in the longitudinal structures but also in front of $\left(\delta_{\mu \nu}-\right.$ $\left.n_{\mu} n_{\nu}\right)$. It turns out that the singular structure is due to the Dirac strings, which are directed along vector $n_{\mu}$ entering the Zwanziger Lagrangian (6). Note that Dirac strings become short strings or ANO strings in the dual AHM.

Although expression (811) for the gluon propagator is exact, it contains an unknown function $D^{\Sigma}\left(p^{2}\right)$. The infrared behavior of this function however can be qualitatively understood on physical grounds. Indeed, consider the string part (11) of the gluon propagator (8). This part corresponds to the following process: the quark emits a gauge boson $A$ which transforms to a dual gauge boson $B$ via coupling $\langle A \cdot B\rangle$ which exists in the Zwanziger Lagrangian (6). The dual gauge boson transforms back to the gauge boson after scattering on a closed ANO string world sheet $\Sigma$. The intermediate string state is described by the function $D^{\Sigma}\left(p^{2}\right)$ and this state can be considered as a glueball state with the photon quantum numbers $1^{-}$.

The behavior of the function $D^{\Sigma}\left(p^{2}\right)$ in the infrared region, $p \rightarrow 0$, can be estimated as $D^{\Sigma}\left(p^{2}\right)=\frac{C}{p^{2}+M_{\mathrm{gl}}^{2}}+\ldots$, where $C$ is a dimensionless parameter and $M_{\mathrm{gl}}$ is the mass of the lowest $1^{-}$glueball. The dots denote the contributions of heavier states. Thus, according to eqs. (9, 10) in the infrared limit, $p \rightarrow 0$

$$
\begin{array}{r}
F^{\mathrm{IR}}\left(p^{2}\right)=\frac{1}{m_{V}^{2}}+\frac{C}{M_{\mathrm{gl}}^{2}}+O\left(p^{2}\right) \\
G^{\mathrm{IR}}\left(p^{2}\right)=-1+O\left(p^{2}\right)
\end{array}
$$

Note that neither double, $1 /\left(p^{2}\right)^{2}$, nor ordinary, $1 / p^{2}$, pole are exhibited by the gluon propagator (8-12) in the infrared limit. There are gauge dependent $1 /(p \cdot n)$ singularities which reflect the presence of Dirac strings. Therefore our gauge boson propagator does not vanish in the infrared either. In view of this, the vanishing of the propagator at $p \rightarrow 0$ predicted in [9] seems to be specific for the special gauge choice in QCD which leads to a nontrivial Fundamental Modular Region.

\section{Relation of potential and propagator, violation of the operator product expan- sion.}

As it is mentioned above, the existence of short strings is manifested also through breaking of the 
standard OPE. The physical reason of this breaking is that strings have extra dimensionality compared to particles, they are not point-like objects. The another manifestation of this problem is the violation of the standard relation between the potential and the propagator. Let us try to reconstruct the classical Coulomb potential between (heavy) electric charge-anticharge and between magnetic charge-anticharge in the dual AHM for $r \ll m_{V}^{-1}, m_{H}^{-1}$. For magnetic charges the potential can be reconstructed in the standard way from the propagator $\tilde{D}_{\mu \nu}\left(q^{2}\right)$ in the momentum space,

$V(r)=\int d^{3} q e^{i \mathbf{q} \cdot \mathbf{r}} \tilde{D}_{00}\left(\mathbf{q}^{2}\right)$.

In order to have the standard notations we use here $q$ in the Minkovsky region, $q^{2}=-p^{2}$ (cf. eq. (8) - (11)). For $q$ in Euclidean region and much larger than the mass parameters, the propagator $\tilde{D}_{\mu \nu}\left(q^{2}\right)$ can be evaluated by using the OPE. Restriction to the classical approximation implies that loop contributions are not included. However, vacuum fields which are soft on the scale of $\mathbf{q}^{2}$ can be consistently accounted for in this way (for a review of see, e.g., 18]). Modulus longitudinal terms, we have:

$$
\begin{array}{r}
\tilde{D}_{\mu \nu}\left(q^{2}\right)=\delta_{\mu \nu}\left(\frac{1}{q^{2}}+\frac{1}{q^{2}} g^{2}\left\langle\Phi^{2}\right\rangle \frac{1}{q^{2}}+\right. \\
\left.\frac{1}{q^{2}} g^{2}\left\langle\Phi^{2}\right\rangle \frac{1}{q^{2}} g^{2}\left\langle\Phi^{2}\right\rangle \frac{1}{q^{2}}+\ldots\right)=\frac{\delta_{\mu \nu}}{q^{2}-m_{V}^{2}} .
\end{array}
$$

Thus, one uses first the general OPE assuming $\left|q^{2}\right| \gg g^{2} \Phi^{2}$ then substitutes the vacuum expectation of the Higgs field $\Phi$ and upon summation of the whole series of the power corrections reproduces the propagator of a massive particle $\left(<\Phi^{2}>=\eta^{2}, m_{V}^{2}=g^{2} \eta^{2}\right)$. This propagator according to (13) corresponds to Yukawa potential, or to Coulomb potential at small distances.

The photon exchange between electric charges (analogues of quarks) corresponds to propagator (8). At the classical level we should neglect the contribution of virtual strings, $D^{\Sigma}\left(p^{2}\right)=0$, and:

$D_{\mu \nu}\left(q^{2}\right)=\frac{1}{q^{2}-m_{V}^{2}}\left(\delta_{\mu \nu}-\frac{1}{(q n)}\left(q_{\mu} n_{\nu}+q_{\nu} n_{\mu}\right)\right.$

$$
\left.+\frac{q_{\mu} q_{\nu}}{(q n)^{2}}+\frac{m_{V}^{2}}{(q n)^{2}}\left(\delta_{\mu \nu} n^{2}-n_{\mu} n_{\nu}\right)\right) .
$$

If the potential energy is given by the Fourier transform of (15) then its dependence on $n_{\mu}$ is explicit. Thus it is impossible to get the Coulomb potential by the Fourier transform (13), we addressed this problem in Ref. 19].

Note that Eq. (15) immediately implies that the standard OPE does not work any longer on the level of $q^{-2}$ corrections. Indeed, choosing $q^{2}$ large and negative does not guarantee now that the $m_{V}^{2}$ correction is small since the factor $(q n)^{2}$ in the denominator may become zero.

The reason for the breaking of the standard $\mathrm{OPE}$ is that even at short distances the dynamics of the short strings should be accounted for explicitly. In particular, in the classical approximation the string lies along the straight line connecting the magnetic charges and affects the solution through the corresponding boundary condition, see above. The OPE is valid as far as the probe particles do not change the vacuum fields drastically and the unperturbed vacuum fields are a reasonable zero-order approximation. In our case, however, the Higgs field is brought down to zero along the string and this is a nonperturbative effect.

\section{Short strings in QCD}

Now we briefly review the possible manifestations of short strings in numerical simulations of lattice gluodynamics.

(i) The lattice simulation 32] do not show any change in the slope of the full $Q \bar{Q}$ potential as the distances are changed from the largest to the smallest ones where Coulombic part becomes dominant. Thus eq. (4) is presumably satisfied. Moreover, it is known from phenomenological analysis and from the calculations on the lattice 20 22] that the realistic QCD corresponds to the case $\kappa \approx 1$ where $\kappa=m_{H} / m_{V}$. It is remarkable that, as is mentioned above, the AHM in the classical approximation also results in the relation (4) for $\kappa \approx 1$.

(ii) Addressing the question of linear corrections at small distances one tries to explicitly substruct the perturbative contribution from the full 
$Q \bar{Q}$ potential. It appears that the remaining part is well parameterized by linear function and the extrapolation of the lattice data to very small distances gives large value for $\sigma_{0}, \sigma_{0} \approx 6 \sigma_{\infty}$ 33.

(iii) There exist lattice measurements of fine splitting of the $Q \bar{Q}$ levels as a function of the heavy quark mass. The Voloshin-Leutwyler 23] picture results in a particular pattern of the mass dependence of this splitting. Moreover, these predictions are very different from the predictions based on adding a linear part to the Coulomb potential (Buchmuller-Tye potential [24]). The results of most advanced measurements of this type [25] are in favor of the linear (3 3 - 4 ) correction to the potential at short distances.

(iv) There is an evidence that the nonperturbative fluctuations on the lattice responsible for the confinement can be identified with the so called $\mathrm{P}$ vortices [26]. If one measures the quark potential due only to these vortices, the numerical results seem to indicate that the slope of the potential is the same at large and at small distances, see [27.

$(v)$ The dimensional analysis show that the linear correction at short distances to the potential $V(r)$ produced by short string corresponds to $1 / Q^{2}$ correction in OPE. The standard leading correction is only $1 / Q^{4}$, which corresponds to the operator $\left\langle\operatorname{Tr} F_{\mu \nu}^{2}>\right.$. Numerical data for the expectation value of the plaquette minus perturbation theory contribution show $1 / Q^{2}$ behavior 28 .

At present the systematical errors in numerical calculations are large, especially the subtraction of perturbation theory contribution in items (ii) and $(v)$ is not a well established procedure, nevertheless it seems that there are several independent facts showing the existence of the short strings in lattice gluodynamics.

Now we discuss two effects which can be manifestation of the short strings in QCD.

(i) Analytical studies of the Bethe-Salpeter equation and comparison of the results with the charmonium spectrum data favor a nonvanishing linear correction to the potential at short distances [29].

(ii) The most interesting manifestation of short strings is $1 / Q^{2}$ corrections to the standard OPE for current-current correlation function $\Pi\left(Q^{2}\right)$
3, 30, 4. It is impossible to calculate the coefficient of $1 / Q^{2}$ corrections, and in ref. [30] it was suggested to simulate this correction by tahyonic gluon mass. The Yukawa potential with an imaginary mass has the linear attractive piece at small distances as it is induced by short strings. The use of the gluon propagator with the imaginary gluon mass $\left(m_{g}^{2}=-0.5 \mathrm{Gev}^{2}\right)$ unexpectively well explains the different behavior of $\Pi\left(Q^{2}\right)$ in different channels. It is very important to perform the accurate calculations of various correlators $\Pi\left(Q^{2}\right)$ on the lattice to check the model. There are theoretical schemes [31] which imitate the tachyonic gluon mass.

\section{Acknowledgements}

We are acknowledging thankfully discussions with A. Leonidov, V.A. Novikov, L. Stodolsky, A.I. Vainshtein, M.I. Vysotsky. Work of M.N.C., F.V.G. and M.I.P. was partially supported by grants INTAS-RFBR-95-0681, RFBR 99-01230a and INTAS 96-370.

\section{REFERENCES}

1. F.V.Gubarev, M.I.Polikarpov, V.I.Zakharov, hep-th/9812030, ITEP-TH-73/98

2. Z.F.Ezawa, A.Iwazaki, Phys.Rev. D25 (1982) 2681 ;

T.Suzuki, I.Yotsuyanagi, Phys. Rev., D42 (1990) 4257.

3. R.Akhoury, V.I.Zakharov, Phys. Lett. B438 (1998) 165.

4. F.V.Gubarev, M.I.Polikarpov, V.I.Zakharov, hep-ph/9908292, ITEP-TH-36-99; hep-ph/9908293, ITEP-TH-37-99.

5. G. 't Hooft, Nucl. Phys. B190 (1981) 455.

6. J.W.Alcock, M.J.Burfitt, W.N.Cottingham, Nucl. Phys. B226 (1983) 299.

7. G.B.West, Phys. Lett. 115B (1982) 468.

8. M.Baker, J.S.Ball and F.Zachariasen, Phys. Rev. D37 (1988) 1036; Erratum-ibid. D37 (1988) 3785.

9. V.N.Gribov, Nucl. Phys. B139 (1978) 1; D.Zwanziger, Nucl. Phys. B364 (1991) 127.

10. U.Häbel et al., Z.Phys. A 336 (1990) 423; L. von Smekal, A.Hauck, R.Alkofer, Phys. 
Rev. Lett. 79 (1997) 3591; Ann. Phys. 267 (1998) 1;

D.Atkinson, J.C.R.Bloch, Mod. Phys. Lett A13 (1998) 1055.

11. M.Baker, J.S.Ball, F.Zachariasen, Nucl. Phys. B186 (1981) 531;

N.Brown, M.R.Pennington, Phys. Rev. D39 (1989) 2723;

A.I.Alekseev, Phys. Lett. B344 (1995) 325;

F.T.Hawes, P.Maris, C.D.Roberts, Phys. Lett. B440 (1998) 353.

12. C.Bernard, C.Parrinello, A.Soni, Phys. Rev. D49 (1994) 1585;

H.Nakajima, S.Furui, Nucl. Phys. Proc. Suppl. 73 (1999) 635-637;

A.Cucchieri, Phys. Lett. B422 (1998) 233;

D.B.Leinweber et al., hep-lat/9811027, ADP98-72/T339;

J.P.Ma, hep-lat/9903009, AS-ITP-99-07.

13. D.Zwanziger, Phys. Rev. D3 (1971) 343;

R.A.Brandt, F.Neri, D.Zwanziger, Phys. Rev. D19 (1979) 1153;

M.Blagojević, P.Senjanović, Phys. Rep. 157 (1988) 233.

14. A.P.Balachandran, H.Rupertsberger, J.Schechter, Phys. Rev. D11 (1975) 2260;

T.Suzuki, Prog. Theor. Phys. 81 (1989) 752.

15. S.Maedan, T.Suzuki, Prog. Theor. Phys. 81 (1989) 229.

16. P.Orland, Nucl. Phys. B428 (1994) 221;

M.Sato, S.Yahikozawa, Nucl. Phys. B436 (1995) 100;

E.T.Akhmedov et al., Phys. Rev. D53 (1996) 2087;

M.I.Polikarpov, U.-J.Wiese, M.A.Zubkov, Phys. Lett. 309B (1993) 133.

17. M.N.Chernodub, M.I.Polikarpov, V.I.Zakharov, Phys. Lett. B457 (1999) 147.

18. V.A.Novikov et.al., Fortsch. Phys. 32 (1985) 585 .

19. F.V.Gubarev, M.I.Polikarpov, V.I.Zakharov, Phys. Lett. B438 (1998) 147.

20. T.Suzuki, Progr. Theor. Phys. 80 (1988) 929; ibid 81 (1989) 752.

21. M.Baker, J.S.Ball, F.Zachariasen, Phys. Rev. D41 (1990) 2612;

V.Singh, R.Haymaker, D.Browne, Phys. Rev. D47 (1993) 1715;
Y.Matsubara, S.Ejiri, T.Suzuki, Nucl. Phys. Proc. Suppl. 34 (1994) 176;

S.Kato et al., Nucl. Phys. Proc. Suppl. 63 (1998) 471;

H.Suganuma, S.Sasaki, H.Toki, Nucl. Phys. B435 (1995) 207.

22. G.S.Bali, C.Schlichter, K.Schilling, Prog. Theor. Phys. Suppl. 131 (1998) 645-656.

23. M.B.Voloshin, Nucl. Phys. 154 (1979) 365;

H.Leutwyler, Phys. Lett. B98 (1981) 447.

24. W.Buchmuller, S.H.H.Tye, Phys. Rev. D24 (1981) 132.

25. J.Fingberg, Nucl. Phys. Proc. Suppl. $\mathbf{7 3}$ (1999) 348-350.

26. M.Faber, J.Greensite, S.Olejnik, JHEP 9901 (1999) 008.

27. L. Del Debbio, M. Faber, J. Greensite, S. Olejnik, Nucl. Phys. Proc. Suppl. 53 (1997) 141.

28. G. Burgio, F. Di Renzo, G. Marchesini, E. Onofri Nucl. Phys. Proc. Suppl. 63 (1998) 805.

29. A.M.Badalian, V.L.Morgunov, hep-ph/990143d, ITEP-PH-8-1998.

30. K.G.Chetyrkin, S.Narison, V.I.Zakharov, Nucl. Phys., B550 (1999) 353.

31. Yu.A.Simonov, JETP Lett. 69 (1999) 505; hep-ph/9902233;

S.J.Huber, M.Reuter, M.G.Schmidt, hepph/9906358, HD-THEP/99-19, MZ-TH/9922.

32. G.S.Bali, K.Schilling, A.Wachter, Phys. Rev. D55 (1997) 5309.

33. G.S.Bali, Phys. Lett. B460 (1999) 170. 